Note

\title{
Synthesis of the pentasaccharide repeating unit of Escherichia coli 0128 antigen
}

\author{
Xun $\mathrm{Lv}^{\mathrm{a}, \mathrm{b}}$, Shuihong Cheng ${ }^{\mathrm{c}}$, Guoha Wei ${ }^{\mathrm{a}}$, Yuguo $\mathrm{Du}^{\mathrm{a}, \mathrm{b}, *}$ \\ a State Key Laboratory of Environmental Chemistry and Ecotoxicology, Research Center for Eco-Environmental Sciences, Chinese Academy of Sciences, Beijing 100085, China \\ ${ }^{\mathrm{b}}$ College of Chemistry and Chemical Engineering, Graduate University of Chinese Academy of Sciences, Beijing 100049, China \\ ${ }^{\mathrm{c}}$ CAS Key Laboratory of Pathogenic Microbiology and Immunology, Institute of Microbiology, Chinese Academy of Sciences, Beijing 100101, China
}

\section{A R T I C L E I N F O}

\section{Article history:}

Received 15 June 2010

Received in revised form 9 July 2010

Accepted 14 July 2010

Available online 21 July 2010

\section{Keywords:}

Glycosylation

Escherichia coli 0128 antigen

Oligosaccharides

\begin{abstract}
A B S T R A C T
A pentasaccharide, 4-methoxyphenyl 2 -acetamido-2-deoxy- $\beta$-D-galactopyranosyl-( $1 \rightarrow 4)-\alpha$-D-galactopyranosyl-( $1 \rightarrow 3)$-2-acetamido-2-deoxy- $\beta$-D-galactopyranosyl- $(1 \rightarrow 6)$-[ $\alpha$-L-fucopyranosyl-( $1 \rightarrow 2)]-\beta$-Dgalactopyranoside (1), representing the repeating unit of Escherichia coli 0128 antigen, was successfully prepared in $23 \%$ overall yield via a convergent ' $2+3$ ' glycosylation strategy.
\end{abstract}

(c) 2010 Elsevier Ltd. All rights reserved.
The O-antigens (O-specific polysaccharides) are the exposed part of the lipopolysaccharides (LPS), the main outer-membrane component of Gram-negative bacteria. Structural variations of O-antigens contribute to the wide variety of antigenic types of different bacterial species. ${ }^{1}$ O-antigens are furthermore recognized as important virulence factors and have the potential to influence host-pathogen interactions in many ways. ${ }^{2}$ During the investigation on the molecular basis related to infantile diarrhea, E. coli 0128 antigen has been isolated and characterized from enteropathogenic E. coli strains, one of the major species associated with diarrhea patients worldwide. ${ }^{3}$ The primary structure of the $O$-antigen repeating unit of $E$. coli 0128 has been established ${ }^{4}$ as $\left.\rightarrow 3\right)$ - $\beta$-D-GalNAc- $(1 \rightarrow 4)-\alpha-D-G a l-$ $(1 \rightarrow 3)-\beta-D-G a l N A c-(1 \rightarrow 6)-[\alpha-L-F u c-(1 \rightarrow 2)]-\beta-D-G a l-(1 \rightarrow$, a pentasaccharide (Fig. 1 ) in which [ $\alpha$-L-Fuc- $(1 \rightarrow 2)]-\beta-D-G a l$ was proposed to be the immunodominant part. Due to frequently occurring antibiotic-resistant pathogens, there are stringent requirements in developing potent vaccines against infectious diseases. Vaccines consisting of carbohydrates coupled to a carrier protein have been found to be effective for the prevention of bacterial invasive diseases. ${ }^{5,6}$ Thus, providing structurally defined glycoconjugates via synthetic carbohydrate antigens would be one of the attractive strategies in healing infectious diseases. ${ }^{7,8}$ As a collaborative project, we envisioned that the efficient synthesis of the pentasaccharide repeating unit of the 0-128 antigen would pave way for the development of a well-structured glycoconjugate vaccine against infantile diarrhea E. coli 0128 . We herein report the synthesis of the core pentasaccharide using a convergent ' $2+3$ ' glycosylation strategy.

\footnotetext{
* Corresponding author. Tel./fax: +86 1062849126.

E-mail address: duyuguo@rcees.ac.cn (Y. Du).
}

In our previous work, we found that using a $\beta$-( $1 \rightarrow 4)$-linked disaccharide donor might generate an $\alpha$-glycosidic product under proper glycosylation conditions. ${ }^{9}$ Accordingly, E. coli 0128 repeating pentasaccharide could be disconnected into a disaccharide donor 2 , in which the 2-OH was blocked with a non-neighboring participation group such as benzyl, and a trisaccharide acceptor 3. Both $\mathbf{2}$ and $\mathbf{3}$ could be assembled from known or commercially available monosaccharide building blocks 4-8 (Scheme 1).

In the preparation of disaccharide donor $\mathbf{2}$, we first tried the coupling reaction between compounds $\mathbf{4}^{10}$ and $5^{11}$ with the traditional method. ${ }^{12}$ Unfortunately, we observed a significant amount of byproduct formed via thio-group transfer ${ }^{13-15}$ from 5 to 4 . We thus turned our attention to the 'inverse procedure'16 under low temperature (Scheme 2), in which donor 4 in $\mathrm{CH}_{2} \mathrm{Cl}_{2}$ was added into a pre-cooled solution of acceptor $\mathbf{5}$ and the promoter, trimethylsilyl trifluoromethanesulfonate (TMSOTf), in $\mathrm{CH}_{2} \mathrm{Cl}_{2}$ at $-15^{\circ} \mathrm{C}$. As expected, synthesis of disaccharide $\mathbf{2}$ was successfully achieved in

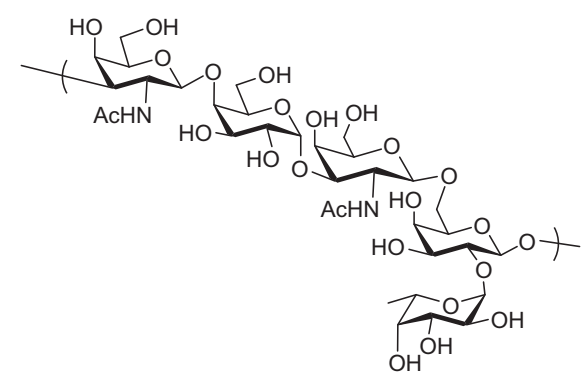

Figure 1. Structure of E. coli 0128 antigen. 

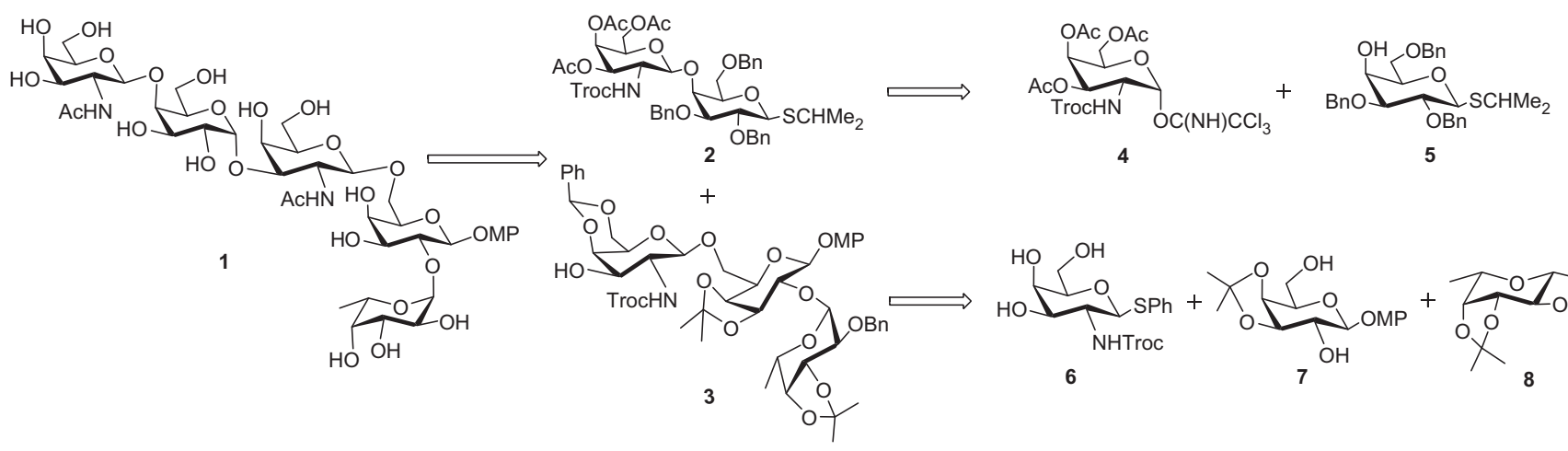

Scheme 1. Retrosynthetic analysis of E. coli 0128 pentasaccharide.

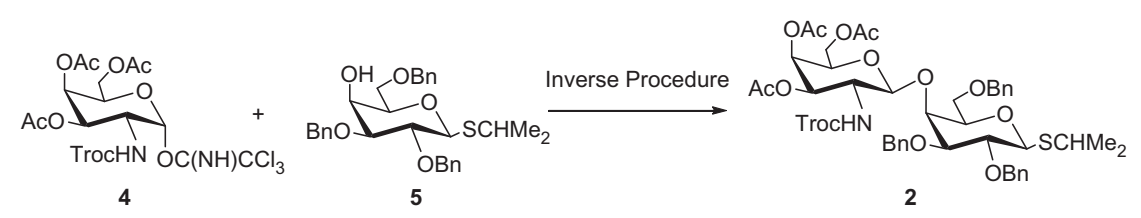

Scheme 2. Synthesis of disaccharide donor 2.

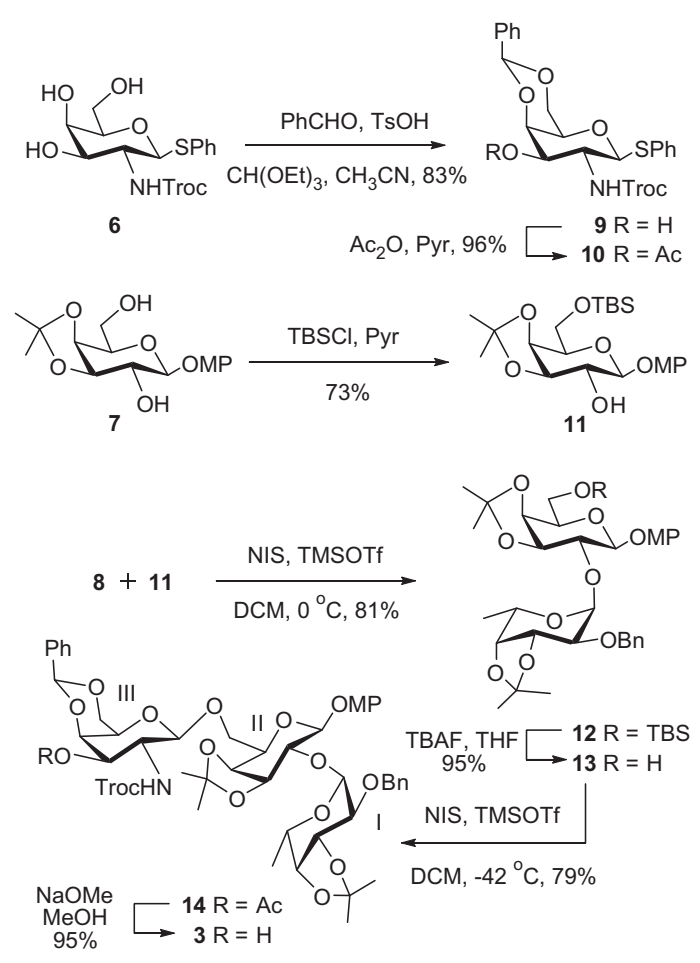

Scheme 3. Synthesis of trisaccharide acceptor 3.

an acceptable isolated yield of $73 \%$, in addition to its $6 \%$ of the $\alpha$ isomer.

As depicted in Scheme 3, treatment of phenyl 2-deoxy-2-(2,2,2trichloroethoxycarbonylamino)-1-thio- $\beta$-D-galactopyranoside $(6)^{17}$ with $\mathrm{PhCHO}$, trimethyl orthoformate $\left[\mathrm{CH}(\mathrm{OEt})_{3}\right]$, and TsOH in acetonitrile gave phenyl 4,6-O-benzylidene-2-deoxy-2-(2,2,2-trichloroethoxycarbonylamino)-1-thio- $\beta$-D-galactopyranoside (9), which was then acetylated with $\mathrm{Ac}_{2} \mathrm{O}$ in pyridine to afford $\mathbf{1 0}$ in $79 \%$ yield over two steps. To differentiate the $2-\mathrm{OH}$ from the $6-\mathrm{OH}$ of 4-methoxyphenyl 3,4-O-isopropylidene- $\beta$-D-galactopyranoside (7), commercially available $\mathbf{7}$ was treated with $\mathrm{TBSCl}$ in pyridine at
$0{ }^{\circ} \mathrm{C}$, generating 4-methoxyphenyl 6-O-tert-butyldimethylsilyl3,4-O-isopropylidene- $\beta$-D-galactopyranoside (11). Condensation of ethyl 2-O-benzyl-3,4-O-isopropylidene- $\beta$-L-fucopyranoside $(\mathbf{8})^{18}$ and 11 in the presence of $\mathrm{N}$-iodosuccinimide (NIS) and TMSOTf at $0{ }^{\circ} \mathrm{C}$ in $\mathrm{CH}_{2} \mathrm{Cl}_{2}$ gave disaccharide $12(81 \%)$. Desilylation of 12 with tetrabutylammonium fluoride (TBAF) in THF yielded the disaccharide acceptor 13 , which was further coupled with $\mathbf{1 0}$, under the same reaction conditions as described in the preparation of 12, to afford trisaccharide 14. Deacetylation of 14 with $\mathrm{NaOMe}$ in $\mathrm{MeOH}$ furnished trisaccharide acceptor $\mathbf{3}$ in a yield of $70 \%$ over three steps.

In order to improve the efficiency in making trisaccharide derivative 14, we also investigated one-pot glycosylation as shown in Scheme 4. Taking advantage of different reactivity between the primary and secondary alcohols of acceptor $\mathbf{7}$, donor $\mathbf{1 0}$ was first added to a pre-cooled $\left(-42{ }^{\circ} \mathrm{C}\right)$ solution of 7 in $\mathrm{CH}_{2} \mathrm{Cl}_{2}$ in the presence of co-catalysts NIS-TMSOTf. An additional amount of $\mathbf{1 0}$ was added on occasion to push the reaction to completion. When acceptor $\mathbf{7}$ was completely consumed, the second glycosyl donor 8 was added to the same reaction system at $-15^{\circ} \mathrm{C}$, together with another portion of NIS-TMSOTf. Compound 14 was eventually obtained in $28-43 \%$ isolated yields. However, this method provided unreliable yield, and the purification step was also tedious. We thus prepared 14 step-by-step as shown in Scheme 3.

With both disaccharide donor and trisaccharide acceptor in hands, we next explored the condensation of $\mathbf{2}$ and $\mathbf{3}$ catalyzed by NIS and TMSOTf in anhyd $\mathrm{CH}_{2} \mathrm{Cl}_{2}$ at $-20{ }^{\circ} \mathrm{C}$ (Scheme 5). As we expected, pentasaccharide $\mathbf{1 5}$ was formed with the $\alpha$-glycoside as the major product in $84 \%$ isolated yield. Based on COSY spectra of 15, the chemical shifts corresponding to the new glycosidic bond showed at $5.25 \mathrm{ppm}\left({ }^{1} \mathrm{H} \mathrm{NMR}, \mathrm{H}-1^{\mathrm{IV}}, J=3.0 \mathrm{~Hz}\right)$ and $95.45 \mathrm{ppm}$
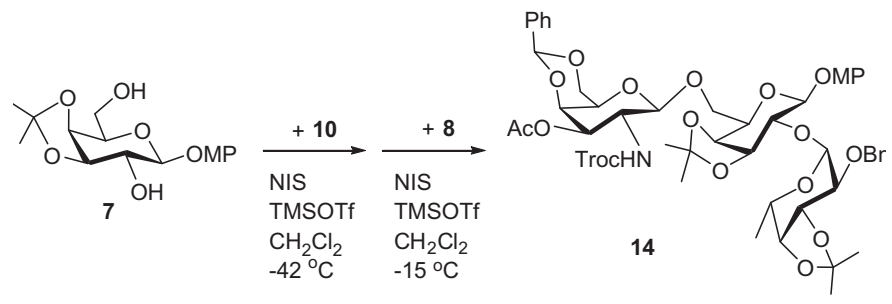

Scheme 4. One-pot synthesis of trisaccharide 14. 


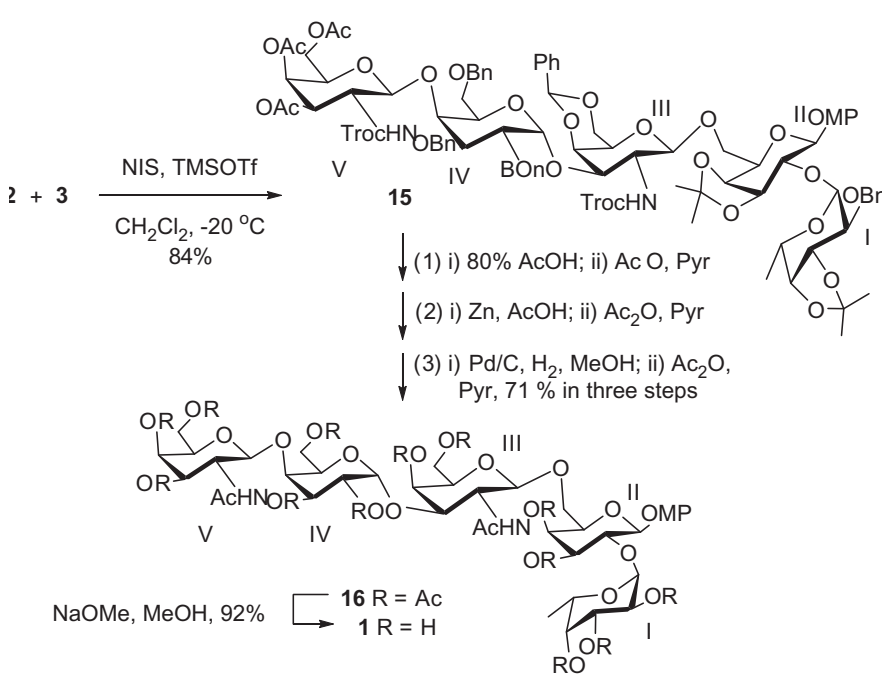

Scheme 5. Synthesis of pentasaccharide 1.

$\left({ }^{13} \mathrm{C} \mathrm{NMR}, \mathrm{C}-1^{\mathrm{IV}}\right)$, respectively. Deacetalation of 15 with $80 \% \mathrm{AcOH}$ at $85^{\circ} \mathrm{C}$, followed by removal of Troc with zinc in $\mathrm{AcOH}$, debenzylation with $10 \% \mathrm{Pd} / \mathrm{C}$ under $\mathrm{H}_{2}$ pressure, and acetylation of the residue with $\mathrm{Ac}_{2} \mathrm{O}$ in pyridine, generated fully acetylated pentasaccharide $\mathbf{1 6}$ in $71 \%$ yield from $15 .{ }^{19}$ In the COSY spectra of $\mathbf{1 6}$, a broad singlet at $5.13 \mathrm{ppm}(J<3.0 \mathrm{~Hz})$ and the peak at $94.6 \mathrm{ppm}$ represent $\mathrm{H}-1^{\mathrm{IV}}$ and $\mathrm{C}-1^{\mathrm{IV}}$, respectively, further confirming its structural assignment. Saponification of $\mathbf{1 6}$ with $\mathrm{NaOMe}$ in $\mathrm{MeOH}$ quantitatively furnished the desired pentasaccharide $\mathbf{1}$.

In summary, we have developed a practical route for the synthesis of the pentasaccharide repeating unit of $E$. coli 0128 antigen via a facile $\alpha$-bond formation. This successful example supports the argument that remote controls affect the stereoselectivity in the construction of an oligosaccharide, leading to a new glycosyl bond with an opposite configuration to that of the established bond in either the donor or acceptor. ${ }^{20}$ Application of this pentasaccharide in vaccine development is currently under exploration.

\section{Experimental}

\subsection{General methods}

Optical rotations were determined at $25^{\circ} \mathrm{C}$ with a Perkin-Elmer Model 241-Mc automatic polarimeter. ${ }^{1} \mathrm{H}$ NMR, ${ }^{13} \mathrm{C}$ NMR, and COSY spectra were recorded with Bruker ARX 500 (or 400) spectrometers for solutions in $\mathrm{CDCl}_{3}$ or $\mathrm{D}_{2} \mathrm{O}$. Chemical shifts are given in ppm downfield from internal $\mathrm{Me}_{4} \mathrm{Si}$. MALDI-TOF mass spectra (MALDITOFMS) were measured using $\alpha$-cyano-4-hydroxycinnamic acid (CCA) as the matrix. Thin-layer chromatography (TLC) was performed on silica gel $\mathrm{HF}_{254}$ with detection by charring with $30 \%$ (v/v) $\mathrm{H}_{2} \mathrm{SO}_{4}$ in $\mathrm{MeOH}$ or in some cases by UV detection. Column chromatography was conducted by elution of a silica gel column (100-200 mesh) with EtOAc-petroleum ether $\left(60-90^{\circ} \mathrm{C}\right)$ as the eluent, or a column of Bio-Gel P2 with water as the eluent. Solutions were concentrated at $<50{ }^{\circ} \mathrm{C}$ under reduced pressure.

\subsection{Isopropyl 3,4,6-tri-0-acetyl-4,6-0-benzylidene-2-deoxy-2- $(2,2,2$-trichloroethoxycarbonylamino)- $\beta$-D-galactopyranosyl- $(1 \rightarrow 4)$-2,3,6-tri-O-benzyl-1-thio- $\beta$-D-galactopyranoside (2)}

To a mixture of 5 (508 mg, $1.0 \mathrm{mmol}$ ) and 4 Å molecular sieves in anhyd $\mathrm{CH}_{2} \mathrm{Cl}_{2}(5 \mathrm{~mL})$ was added TMSOTf $(22 \mu \mathrm{L}, 0.12 \mathrm{mmol})$ at $-42{ }^{\circ} \mathrm{C}$. The mixture was stirred under these conditions for $10 \mathrm{~min}$ with $\mathrm{N}_{2}$ protection, and then a solution of $4(750 \mathrm{mg}, 1.2 \mathrm{mmol})$ in $\mathrm{CH}_{2} \mathrm{Cl}_{2}(10 \mathrm{~mL})$ was added drop-by-drop. The reaction was moni- tored by TLC until compound $\mathbf{5}$ disappeared. The mixture was neutralized with $\mathrm{Et}_{3} \mathrm{~N}$, and filtered, and the filtrate was concentrated. Column chromatography ( $4: 1$ petroleum ether-EtOAc) of the residue gave compound 2 (709 $\mathrm{mg}, 73 \%)$ as a syrup: $[\alpha]_{\mathrm{D}}^{25}+86\left(c 1, \mathrm{CHCl}_{3}\right)$; ${ }^{1} \mathrm{H}$ NMR $\left(500 \mathrm{MHz}, \mathrm{CDCl}_{3}\right): \delta 7.26-7.42(\mathrm{~m}, 15 \mathrm{H}, \mathrm{Ph}), 5.62(\mathrm{~d}, 1 \mathrm{H}, J$ $5.2 \mathrm{~Hz}, \mathrm{NH}$ ), 5.26 (d, $\left.1 \mathrm{H}, J 2.4 \mathrm{~Hz}, \mathrm{H}-4^{\prime}\right), 4.92$ (d, $1 \mathrm{H}, J 8.8 \mathrm{~Hz}, \mathrm{H}-1$ ), 4.88-4.91 (m, 2H), 4.79 (d, 1H, J 7.6 Hz, H-1'), 4.49-4.69 (m, 6H), $4.40(\mathrm{~d}, 1 \mathrm{H}, J 11.5 \mathrm{~Hz}), 4.00-4.11(\mathrm{~m}, 3 \mathrm{H}), 4.92-4.94(\mathrm{~m}, 1 \mathrm{H}), 3.56-$ $3.77(\mathrm{~m}, 6 \mathrm{H}), 3.22-3.24(\mathrm{~m}, 1 \mathrm{H}, \mathrm{SCH}), 2.14,1.99,1.93(3 \mathrm{~s}, 9 \mathrm{H}, 3 \mathrm{Ac})$, 0.85-0.88 (m, $\left.6 \mathrm{H}, \mathrm{CH}\left(\mathrm{CH}_{3}\right)_{2}\right) .{ }^{13} \mathrm{C} \mathrm{NMR}\left(125 \mathrm{MHz}, \mathrm{CDCl}_{3}\right): \delta 170.3$, 170.1, 170.0, 154.1, 138.1, 137.1, 129.0, 128.7, 128.4, 128.2, 127.9, 127.7, 127.6, 101.9, 95.7, 84.7, 83.5, 78.9, 77.3, 77.0, 76.9, 76.7, 76.0, 75.7, 74.4, 74.3, 73.5, 71.8, 70.8, 69.2, 66.5, 61.1, 52.6, 35.3, 23.9, 23.8, 22.6, 20.6, 20.5. Anal. Calcd for $\mathrm{C}_{45} \mathrm{H}_{54} \mathrm{Cl}_{3} \mathrm{NO}_{14} \mathrm{~S}$ : C, 55.64; H, 5.60. Found: C, 55.49; H, 5.50.

\subsection{Phenyl 3-0-acetyl-4,6-0-benzylidene-2-deoxy-2-(2,2,2- trichloroethoxycarbonylamino)- $\beta$-D-galactopyranoside (10)}

A mixture of 6 ( $4.5 \mathrm{~g}, 10.0 \mathrm{mmol})$, PhCHO (1.2 mL, $12.0 \mathrm{mmol})$, $\mathrm{CH}(\mathrm{OEt})_{3}(2.0 \mathrm{~mL}, 12.0 \mathrm{mmol})$, and $\mathrm{TsOH}(190 \mathrm{mg}, 1.0 \mathrm{mmol})$ in $\mathrm{CH}_{3} \mathrm{CN}(50 \mathrm{~mL})$ was stirred at $45^{\circ} \mathrm{C}$ for $5 \mathrm{~h}$, at the end of which time TLC indicated that most of compound $\mathbf{6}$ was consumed. The reaction mixture was neutralized with $\mathrm{Et}_{3} \mathrm{~N}$ and concentrated under diminished pressure, and the residue was purified by column chromatography (3:1 petroleum ether-EtOAc) to give 9 as a foamy solid ( $4.47 \mathrm{~g}$, $83 \%)$. A solution of 9 ( $4.27 \mathrm{~g}, 7.98 \mathrm{mmol})$ in $\mathrm{Pyr}(15 \mathrm{~mL})$ and $\mathrm{Ac}_{2} \mathrm{O}$ $(8 \mathrm{~mL})$ was stirred at $\mathrm{rt}$ for $6 \mathrm{~h}$, then concentrated. Column chromatography (4:1 petroleum ether-EtOAc) of the residue gave $\mathbf{1 0}$ $(4.42 \mathrm{~g}, 96 \%)$ as an amorphous solid: $[\alpha]_{\mathrm{D}}^{25}-53\left(\mathrm{c} 1, \mathrm{CHCl}_{3}\right) ;{ }^{1} \mathrm{H}$ $\operatorname{NMR}\left(400 \mathrm{MHz}, \mathrm{CDCl}_{3}\right): \delta 7.25-7.66(\mathrm{~m}, 10 \mathrm{H}, \mathrm{Ph}), 5.55(\mathrm{~s}, 1 \mathrm{H}, \mathrm{PhCH})$, 5.31 (d, 1H, J $10.2 \mathrm{~Hz}, \mathrm{H}-3), 5.11$ (d, 1H, J $8.0 \mathrm{~Hz}, \mathrm{NH}), 5.07$ (d, 1H, J $10.0 \mathrm{~Hz}, \mathrm{H}-1), 4.73-4.75\left(\mathrm{~m}, 2 \mathrm{H}, \mathrm{CH}_{2} \mathrm{CCl}_{3}\right), 4.37$ (d, $1 \mathrm{H}, J 12.3 \mathrm{~Hz}, \mathrm{H}-$ 6a), 4.35 (s, 1H, H-4), 4.03 (d, 1H, J $12.3 \mathrm{~Hz}, \mathrm{H}-6 \mathrm{~b}), 3.90-3.93$ (m, $1 \mathrm{H}, \mathrm{H}-2), 3.60(\mathrm{~s}, 1 \mathrm{H}, \mathrm{H}-5), 2.03$ (s, 3H, Ac). Anal. Calcd for $\mathrm{C}_{24} \mathrm{H}_{24} \mathrm{Cl}_{3} \mathrm{NO}_{7} \mathrm{~S}$ : C, 49.97; H, 4.19. Found: C, 49.81; H, 4.25.

\subsection{4-Methoxyphenyl 6-0-tert-butyldimethylsilyl-3,4-0- isopropylidene- $\beta$-D-galactopyranoside (11)}

To a commercially available compound 7 (3.27 g, $10.0 \mathrm{mmol})$ in pyridine $(25 \mathrm{~mL})$ was added TBSCl $(1.7 \mathrm{~g}, 12.0 \mathrm{mmol})$ at $0{ }^{\circ} \mathrm{C}$. After stirring under these conditions for $3 \mathrm{~h}$, the reaction was quenched by $\mathrm{MeOH}$, and the mixture was concentrated. The residue was diluted with dichloromethane $(50 \mathrm{~mL})$, washed with satd aq $\mathrm{NaHCO}_{3}$, and the organic phase was dried over $\mathrm{Na}_{2} \mathrm{SO}_{4}$ and concentrated. The residue was purified by column chromatography (4:1 petroleum ether-EtOAc) to give $\mathbf{1 1}(3.21 \mathrm{~g}, 73 \%)$ as a syrup: $[\alpha]_{\mathrm{D}}^{25}-28(c 1$, $\left.\mathrm{CHCl}_{3}\right) ;{ }^{1} \mathrm{H}$ NMR $\left(400 \mathrm{MHz}, \mathrm{CDCl}_{3}\right): \delta 7.00,6.79(2 \mathrm{~d}, 2 \times 2 \mathrm{H}, J$ $\left.8.0 \mathrm{~Hz}, \mathrm{CH}_{3} \mathrm{OC}_{6} \mathrm{H}_{4} \mathrm{O}\right), 4.63(\mathrm{~d}, 1 \mathrm{H}, J 8.2 \mathrm{~Hz}, \mathrm{H}-1), 4.22(\mathrm{~d}, 1 \mathrm{H}, J 5.4 \mathrm{~Hz}$, H-4), 4.13 (t, 1H, J 6.6 Hz, H-3), 4.88-4.90 (m, 3H, H-2, H-6a, H-6b), $3.80(\mathrm{t}, 1 \mathrm{H}, J 7.7 \mathrm{~Hz}, \mathrm{H}-5), 3.75\left(\mathrm{~s}, 3 \mathrm{H}, \mathrm{OCH}_{3}\right), 2.76($ br s, $1 \mathrm{H}, \mathrm{OH})$, $1.55,1.35\left(2 \mathrm{~s}, 6 \mathrm{H}, 2 \mathrm{CH}_{3}\right), 0.89,0.06,0.05\left(3 \mathrm{~s}, 15 \mathrm{H}, 5 \mathrm{CH}_{3}\right)$. Anal. Calcd for $\mathrm{C}_{22} \mathrm{H}_{36} \mathrm{O}_{7} \mathrm{Si}$ : C, 59.97; $\mathrm{H}, 8.24$. Found: $\mathrm{C}, 60.21 ; \mathrm{H}, 8.17$.

\subsection{4-Methoxyphenyl 2-O-benzyl-3,4-O-isopropylidene- $\alpha$-L- fucopyranosyl-(1 $\rightarrow 2)-6$-0-tert-butyldimethylsilyl-3,4-0- isopropylidene- $\beta$-D-galactopyranoside (12)}

To a mixture of compounds 8 (406 $\mathrm{mg}, 1.2 \mathrm{mmol}$ ) and $\mathbf{1 1}$ (480 mg, $1.09 \mathrm{mmol})$ in anhyd $\mathrm{CH}_{2} \mathrm{Cl}_{2}(10 \mathrm{~mL})$ were added NIS (405 mg, $1.8 \mathrm{mmol}$ ) and TMSOTf ( $22 \mu \mathrm{L}, 0.12 \mathrm{mmol}$ ) under an $\mathrm{N}_{2}$ atmosphere at $0{ }^{\circ} \mathrm{C}$. The mixture was stirred under these conditions for $30 \mathrm{~min}$, at the end of which time TLC (3:1 petroleum etherEtOAc) indicated that all the starting materials were consumed. The reaction mixture was neutralized with $\mathrm{Et}_{3} \mathrm{~N}$, diluted with $\mathrm{CH}_{2} \mathrm{Cl}_{2}$ 
(10 mL), and washed with aq $\mathrm{Na}_{2} \mathrm{~S}_{2} \mathrm{O}_{3}$. The organic layer was dried over $\mathrm{Na}_{2} \mathrm{SO}_{4}$ and concentrated. Column chromatography (6:1 petroleum ether-EtOAc) of the residue gave disaccharide $12(633 \mathrm{mg}$, $81 \%)$ as a foamy solid: $[\alpha]_{\mathrm{D}}^{25}-81\left(\mathrm{c} 1, \mathrm{CHCl}_{3}\right) ;{ }^{1} \mathrm{H}$ NMR $(400 \mathrm{MHz}$, $\left.\mathrm{CDCl}_{3}\right): \delta 7.28-7.42(\mathrm{~m}, 5 \mathrm{H}, \mathrm{Ph}), 6.92,6.80\left(2 \mathrm{~d}, 2 \times 2 \mathrm{H}, J 9.1 \mathrm{~Hz}, \mathrm{CH}_{3} \mathrm{O}-\right.$ $\left.\mathrm{C}_{6} \mathrm{H}_{4} \mathrm{O}\right), 5.49(\mathrm{~d}, 1 \mathrm{H}, J 3.6 \mathrm{~Hz}, \mathrm{H}-1), 4.82\left(\mathrm{~d}, 1 \mathrm{H}, J 8.2 \mathrm{~Hz}, \mathrm{H}-1^{\prime}\right), 4.78$ (dd, $\left.2 \mathrm{H}, \mathrm{PhCH}_{2}\right), 4.58-4.60(\mathrm{~m}, 1 \mathrm{H}), 4.34\left(\mathrm{t}, 1 \mathrm{H}, J 6.6 \mathrm{~Hz}, \mathrm{H}-3^{\prime}\right), 4.20-4.22$ $(\mathrm{m}, 2 \mathrm{H}), 4.00-4.02(\mathrm{~m}, 2 \mathrm{H}), 3.86-3.89(\mathrm{~m}, 3 \mathrm{H}), 3.76\left(\mathrm{~s}, 3 \mathrm{H}, \mathrm{CH}_{3} \mathrm{OPh}\right)$, 3.53 (dd, $1 \mathrm{H}, J 3.6,8.0 \mathrm{~Hz}, \mathrm{H}-2), 1.57$ (s, 3H, H-6), 1.33-1.56 (2s, $12 \mathrm{H}$, $\left.4 \mathrm{CH}_{3}\right), 0.92\left(\mathrm{~s}, 9 \mathrm{H}, \mathrm{C}\left(\mathrm{CH}_{3}\right)_{3}\right), 0.07\left(2 \mathrm{~s}, 6 \mathrm{H}, 2 \mathrm{CH}_{3}\right.$ of TBS $)$. Anal. Calcd for $\mathrm{C}_{38} \mathrm{H}_{56} \mathrm{O}_{11} \mathrm{Si}$ : C, 63.66; H, 7.87. Found: C, 63.49; H, 7.96.

\subsection{4-Methoxyphenyl 2-0-benzyl-3,4-0-isopropylidene- $\alpha$-L- fucopyranosyl-( $\rightarrow 3)$-3,4-0-isopropylidene- $\beta$-D- galactopyranoside (13)}

A solution of $12(700 \mathrm{mg}, 0.98 \mathrm{mmol})$ and TBAF (523 mg, $2.0 \mathrm{mmol})$ in THF $(15 \mathrm{~mL})$ was stirred at $0{ }^{\circ} \mathrm{C}$ for $1 \mathrm{~h}$, then for a further $4 \mathrm{~h}$ at rt until the starting material was consumed. The reaction mixture was diluted with EtOAc $(10 \mathrm{~mL})$, then washed with aq $\mathrm{NH}_{4} \mathrm{Cl}$ and water, respectively. The water phase was extracted with EtOAc $(3 \times 3 \mathrm{~mL})$, and the combined organic phase was washed with water and brine, dried, filtered, and concentrated to dryness. The residue was purified by silica gel column chromatography (3:1 petroleum ether-EtOAc) to give $13(560 \mathrm{mg}, 95 \%)$ as a foamy solid: $[\alpha]_{\mathrm{D}}^{25}-70\left(\right.$ c $\left.1, \mathrm{CHCl}_{3}\right) ;{ }^{1} \mathrm{H} \mathrm{NMR}\left(400 \mathrm{MHz}, \mathrm{CDCl}_{3}\right): \delta$ 7.28-7.42 (m, 5H, Ph), 6.89, $6.82\left(2 \mathrm{~d}, 2 \times 2 \mathrm{H}, J 9.1 \mathrm{~Hz}, \mathrm{CH}_{3} \mathrm{OC}_{6} \mathrm{H}_{4} \mathrm{O}\right)$, 5.47 (d, 1H, J $3.6 \mathrm{~Hz}, \mathrm{H}-1), 4.89$ (d, $\left.1 \mathrm{H}, J 8.2 \mathrm{~Hz}, \mathrm{H}-1^{\prime}\right), 4.75$ (dd, $2 \mathrm{H}$, $\mathrm{PhCH}_{2}$ ), 4.54-4.56 (m, 1H), 4.38 (t, 1H, J 6.1 Hz, H-3'), 4.24 (dd, 1H, J $\left.5.6,8.0 \mathrm{~Hz}, \mathrm{H}-2^{\prime}\right), 4.24$ (dd, $\left.1 \mathrm{H}, J 1.6,5.6 \mathrm{~Hz}, \mathrm{H}-4\right), 4.00-4.02$ (m, $4 \mathrm{H}), 3.92$ (br s, $1 \mathrm{H}, \mathrm{OH}), 3.84\left(\mathrm{~s}, 3 \mathrm{H}, \mathrm{CH}_{3} \mathrm{OPh}\right), 3.53$ (dd, $1 \mathrm{H}, J$ 3.6, $8.0 \mathrm{~Hz}, \mathrm{H}-2), 2.17\left(\mathrm{~s}, 3 \mathrm{H}, \mathrm{CH}_{3}\right), 1.34-1.38\left(\mathrm{~m}, 12 \mathrm{H}, \mathrm{H}-6\right.$ and $\left.3 \mathrm{CH}_{3}\right)$. Anal. Calcd for $\mathrm{C}_{32} \mathrm{H}_{42} \mathrm{O}_{11}$ : C, 63.77; H, 7.02. Found: $\mathrm{C}, 63.60 ; \mathrm{H}$, 7.11.

1.7. 4-Methoxyphenyl 2-0-benzyl-3,4-O-isopropylidene- $\alpha$-Lfucopyranosyl- $(1 \rightarrow 2)$-[3-0-acetyl-4,6-0-benzylidene-2-deoxy2-(2,2,2-trichloroethoxycarbonylamino)- $\beta$-D-galactopyranosyl$(1 \rightarrow 6)]-3,4-0$-isopropylidene- $\beta$-D-galactopyranoside $(14)$

To a mixture of compounds $\mathbf{1 0}(750 \mathrm{mg}, 1.3 \mathrm{mmol})$ and $\mathbf{1 3}$ (602 $\mathrm{mg}, 1.0 \mathrm{mmol})$ in anhyd $\mathrm{CH}_{2} \mathrm{Cl}_{2}(12 \mathrm{~mL})$ were added NIS (450 mg, $2.0 \mathrm{mmol}$ ) and TMSOTf $(24 \mu \mathrm{L}, 0.13 \mathrm{mmol})$ under an $\mathrm{N}_{2}$ atmosphere at $-42{ }^{\circ} \mathrm{C}$. The mixture was stirred under these conditions for $1.5 \mathrm{~h}$, quenched by $\mathrm{Et}_{3} \mathrm{~N}$, diluted with $\mathrm{CH}_{2} \mathrm{Cl}_{2}$, and washed with aq $\mathrm{Na}_{2} \mathrm{~S}_{2} \mathrm{O}_{3}$. The organic layer was dried over $\mathrm{Na}_{2} \mathrm{SO}_{4}$ and concentrated. The residue was purified by silica gel column chromatography (2:1 petroleum ether-EtOAc) to give compound 14 (845 mg, 79\%) as a white foamy solid: $[\alpha]_{\mathrm{D}}^{25}-27\left(\right.$ c $\left.2, \mathrm{CHCl}_{3}\right) ;{ }^{1} \mathrm{H}$ $\operatorname{NMR}\left(400 \mathrm{MHz}, \mathrm{CDCl}_{3}\right): \delta 7.24-7.52(\mathrm{~m}, 10 \mathrm{H}, \mathrm{Ph}), 6.96,6.89(2 \mathrm{~d}$, $\left.2 \times 2 \mathrm{H}, J 9.1 \mathrm{~Hz}, \mathrm{CH}_{3} \mathrm{OC}_{6} \mathrm{H}_{4} \mathrm{O}\right), 5.51(\mathrm{~s}, 1 \mathrm{H}, \mathrm{CHPh}), 5.45$ (d, $1 \mathrm{H}, J$ $\left.3.6 \mathrm{~Hz}, \mathrm{H}-1^{\mathrm{I}}\right), 4.92\left(\mathrm{~d}, 1 \mathrm{H}, J 8.4 \mathrm{~Hz}, \mathrm{H}-1^{\mathrm{II}}\right), 4.85-4.88(\mathrm{~m}, 1 \mathrm{H}), 4.78$ (dd, $\left.2 \mathrm{H}, \mathrm{PhCH}_{2}\right), 4.50$ (d, $\left.1 \mathrm{H}, J 10.0 \mathrm{~Hz}, \mathrm{H}-1^{\mathrm{III}}\right), 4.52-4.55(\mathrm{~m}, 3 \mathrm{H})$, $4.22-4.35(\mathrm{~m}, 3 \mathrm{H}), 3.93-4.10(\mathrm{~m}, 4 \mathrm{H}), 3.75\left(\mathrm{~s}, 3 \mathrm{H}, \mathrm{CH}_{3} \mathrm{OPh}\right), 3.51-$ $3.53(\mathrm{~m}, 1 \mathrm{H}), 3.41(\mathrm{~s}, 1 \mathrm{H}), 2.06(\mathrm{~s}, 3 \mathrm{H}, \mathrm{Ac}), 1.56,1.38,1.36,1.34$, $1.33\left(5 \mathrm{~s}, 15 \mathrm{H}, \mathrm{H}-6 \mathrm{I}, 4 \mathrm{CH}_{3}\right)$. Anal. Calcd for $\mathrm{C}_{50} \mathrm{H}_{60} \mathrm{Cl}_{13} \mathrm{NO}_{18}$ : C, 56.16; H, 5.66. Found: C, 56.32; H, 5.41.

1.8. 4-Methoxyphenyl 2-0-benzyl-3,4-0-isopropylidene- $\alpha$-Lfucopyranosyl- $(1 \rightarrow 2)$-[4,6-0-benzylidene-2-deoxy-2-(2,2,2trichloroethoxycarbonylamino)- $\beta$-D-galactopyranosyl- $(1 \rightarrow 6)]-$ 3,4-0-isopropylidene- $\beta$-D-galactopyranoside (3)

NaOMe (1 M) was added to a solution of compound 14 (107 mg, $0.1 \mathrm{mmol})$ in $\mathrm{MeOH}(3 \mathrm{~mL})$ at $0{ }^{\circ} \mathrm{C}$ until the $\mathrm{pH}$ of the solution reached 9-10. The reaction mixture was then stirred at $\mathrm{rt}$ for $4 \mathrm{~h}$ and neutralized with Dowex-50 $\left(\mathrm{H}^{+}\right)$ion-exchange resin. The mixture was filtered, the filtrate was concentrated, and the resulting residue was purified by silica gel column chromatography (3:1 petroleum ether-EtOAc) to afford compound 3 (98 $\mathrm{mg}, 95 \%)$ as an amorphous solid: $[\alpha]_{\mathrm{D}}^{25}-51\left(\mathrm{c} 1, \mathrm{CHCl}_{3}\right)$; ${ }^{1} \mathrm{H}$ NMR $(500 \mathrm{MHz}$, $\left.\mathrm{CDCl}_{3}\right): \delta 7.25-7.50(\mathrm{~m}, 10 \mathrm{H}, \mathrm{Ph}), 6.97,6.89(2 \mathrm{~d}, 2 \times 2 \mathrm{H}, 9.0 \mathrm{~Hz}$, $\mathrm{CH}_{3} \mathrm{OC}_{6} \mathrm{H}_{4} \mathrm{O}$ ), $5.54(\mathrm{~s}, 1 \mathrm{H}, \mathrm{CHPh}), 5.45\left(\mathrm{~d}, 1 \mathrm{H}, J 3.5 \mathrm{~Hz}, \mathrm{H}-1^{\mathrm{I}}\right), 4.91$ $\left(\mathrm{d}, 1 \mathrm{H}, J 8.0 \mathrm{~Hz}, \mathrm{H}-1^{\mathrm{II}}\right), 4.78$ (dd, $\left.2 \mathrm{H}, \mathrm{PhCH}_{2}\right), 4.67-4.69(\mathrm{~m}, 2 \mathrm{H})$, 4.24-4.35 (m, 3H), 3.93-4.12 (m, 9H), $3.75\left(\mathrm{~s}, 3 \mathrm{H}, \mathrm{CH}_{3} \mathrm{OPh}\right), 3.51-$ $3.53(\mathrm{~m}, 1 \mathrm{H}), 3.41(\mathrm{~s}, 1 \mathrm{H}), 1.56,1.38,1.36,1.34,1.33(5 \mathrm{~s}, 15 \mathrm{H}, \mathrm{H}-$ $\left.6^{\mathrm{I}}, 4 \mathrm{CH}_{3}\right) .{ }^{13} \mathrm{C}$ NMR $\left(125 \mathrm{MHz}, \mathrm{CDCl}_{3}\right): \delta 155.5,154.9,150.8$, $138.1,137.3,129.2,128.3,128.2,127.9,127.6,126.3,118.1$, 114.9, 110.6, 108.7, 101.3, 100.8, 99.6, 95.5, 79.8, 77.3, 77.0, 76.7, 76.2, 75.9, 75.7, 75.6, 74.9, 74.5, 73.9, 73.6, 71.8, 70.9, 69.0, 68.2, 66.5, 62.8, 55.6, 55.3, 28.2, 27.9, 26.5, 26.4, 16.3. Anal. Calcd for $\mathrm{C}_{48} \mathrm{H}_{58} \mathrm{Cl}_{3} \mathrm{NO}_{17}$ : C, 56.12; H, 5.69. Found: C, 56.34; H, 5.58.

1.9. 4-Methoxyphenyl 3,4,6-tri-0-acetyl-2-deoxy-2-(2,2,2trichloroethoxycarbonylamino)- $\beta$-D-galactopyranosyl- $(1 \rightarrow 4)$ 2,3,6-tri-O-benzyl- $\alpha$-D-galactopyranosyl- $(1 \rightarrow 3)-4,6-0$-benzyl idene-2-deoxy-2-(2,2,2-trichloroethoxycarbonylamino)- $\beta$-Dgalactopyranosyl-( $\rightarrow$ 6)-[2-O-benzyl-3,4-0-isopropylidene$\alpha$-L-fucopyranosyl- $(1 \rightarrow 2)]-3,4-0$-isopropylidene- $\beta$ - $D-$ galactopyranoside (15)

To a mixture of 2 (233 mg, $0.24 \mathrm{mmol}$ ) and 3 (205 mg, $0.2 \mathrm{mmol})$ in anhyd $\mathrm{CH}_{2} \mathrm{Cl}_{2}(10 \mathrm{~mL})$ were added NIS $(81 \mathrm{mg}$, $0.36 \mathrm{mmol})$ and TMSOTf $(4.0 \mu \mathrm{L}, 0.022 \mathrm{mmol})$ under an $\mathrm{N}_{2}$ atmosphere at $-20^{\circ} \mathrm{C}$. The mixture was stirred under these conditions for $1.5 \mathrm{~h}$, quenched by the addition of $\mathrm{Et}_{3} \mathrm{~N}$, diluted with $\mathrm{CH}_{2} \mathrm{Cl}_{2}$ $(20 \mathrm{~mL})$, and washed with aq $\mathrm{Na}_{2} \mathrm{~S}_{2} \mathrm{O}_{3}$. The organic layer was dried over $\mathrm{Na}_{2} \mathrm{SO}_{4}$ and concentrated. The residue was purified by silica gel column chromatography (1.5:1 petroleum ether-EtOAc) to give compound 15 (322 mg, 84\%) as a white foamy solid: $[\alpha]_{\mathrm{D}}^{25}+20$ (c 2, $\left.\mathrm{CHCl}_{3}\right) ;{ }^{1} \mathrm{H} \mathrm{NMR}\left(500 \mathrm{MHz}, \mathrm{CDCl}_{3}\right): \delta 7.02-7.50(\mathrm{~m}, 25 \mathrm{H}, \mathrm{Ph}), 6.86-$ $6.89\left(\mathrm{~m}, 4 \mathrm{H}, \mathrm{CH}_{3} \mathrm{OC}_{6} \mathrm{H}_{4} \mathrm{O}\right), 5.52(\mathrm{~s}, 1 \mathrm{H}, \mathrm{PhCH}), 5.48(\mathrm{~d}, 1 \mathrm{H}, J 3.2 \mathrm{~Hz}$, $\left.\mathrm{H}-1^{\mathrm{I}}\right), 5.43(\mathrm{~d}, 1 \mathrm{H}, J 7.4 \mathrm{~Hz}, \mathrm{NH}), 5.25\left(\mathrm{~d}, 1 \mathrm{H}, J 3.0 \mathrm{~Hz}, \mathrm{H}-1^{\mathrm{IV}}\right), 5.12(\mathrm{~s}$, $1 \mathrm{H}, \mathrm{H}-4^{\mathrm{V}}$ ), 4.88 (d, 2H, J11.0 Hz, $\mathrm{PhCH}_{2}$ ), 4.78-4.81 (m, 3H), 4.67 (d, $\left.1 \mathrm{H}, J 12.2 \mathrm{~Hz}, \mathrm{PhCH}_{2}\right), 4.46-4.58(\mathrm{~m}, 8 \mathrm{H}), 4.33\left(\mathrm{t}, 1 \mathrm{H}, J 6.3 \mathrm{~Hz}, \mathrm{H}-3^{\mathrm{II}}\right)$, $3.90-4.29(\mathrm{~m}, 21 \mathrm{H}), 3.64-3.73(\mathrm{~m}, 6 \mathrm{H}), 3.62-3.64(\mathrm{~m}, 1 \mathrm{H}), 3.54(\mathrm{dd}$, $\left.1 \mathrm{H}, J 3.3,8.1 \mathrm{~Hz}, \mathrm{H}-5^{\mathrm{I}}\right), 3.14(\mathrm{~s}, 1 \mathrm{H}), 2.14,2.03,1.91$ (3s, 9H, 3Ac), $1.59(\mathrm{~s}, 3 \mathrm{H}), 1.37(2 \mathrm{~s}, 12 \mathrm{H}) .{ }^{13} \mathrm{C}$ NMR $\left(125 \mathrm{MHz}, \mathrm{CDCl}_{3}\right): \delta 176.9$, $173.2,172.9,170.0,155.0,154.4,153.8,151.4,138.2,137.9$, $137.6,128.9,128.6,128.2,127.9,127.6,126.4,117.5,114.8$, 110.4, 108.7, 102.0, 101.2, 101.1, 99.4,95.4, 80.0, 78.2, 77.3, 77.0, 76.7, 76.5, 76.3, 76.0, 75.7, 74.6, 74.1, 73.8, 72.5, 71.8, 71.2, 71.0, 70.4, 69.2, 68.8, 68.5, 66.6, 66.3, 62.8, 62.1, 61.4, 60.4, 55.6, 52.5, 39.3 , 37.4, 37.1, 35.9, 34.0, 33.7, 33.4, 32.7, 31.9, 30.0, 29.7, 29.3, 29.1, 28.2, 28.0, 27.4, 27.2, 26.5, 24.8, 24.4, 22.7, 20.7, 19.7, 19.2, 18.3, 16.3, 14.4, 14.1. Anal. Calcd for $\mathrm{C}_{90} \mathrm{H}_{104} \mathrm{Cl}_{6} \mathrm{~N}_{2} \mathrm{O}_{31}$ : C, 56.23; H, 5.45. Found: C, 56.02; H, 5.53.

1.10. 4-Methoxyphenyl 2-acetamido-3,4,6-tri-0-acetyl-2-deoxy$\beta$-D-galactopyranosyl- $(1 \rightarrow 4)-2,3,6$-tri-0-acetyl- $\alpha$-D-galactopyran osyl-(1 $\rightarrow 3)$-2-acetamido-4,6-di-0-acetyl-2-deoxy- $\beta$-D-galacto pyranosyl-( $1 \rightarrow 6)$-[2,3,4-tri- 0 -acetyl- $\alpha$-L-fucopyranosyl-

$(1 \rightarrow 2)]-3,4-d i-0$-acetyl- $\beta$-D-galactopyranoside (16)

Compound 15 (401 mg, $0.21 \mathrm{mmol}$ ) in $80 \%$ aq AcOH $(15 \mathrm{~mL}$ ) was stirred at $85^{\circ} \mathrm{C}$ for $35 \mathrm{~min}$, at the end of which time the mixture was concentrated, and the residue was treated with $\mathrm{Ac}_{2} \mathrm{O}$ $(1 \mathrm{~mL})$ in pyridine $(2 \mathrm{~mL})$ at $\mathrm{rt}$ for $4 \mathrm{~h}$. The mixture was concentrated, and the residue was purified by flash silica gel column chromatography. The white foamy product thus generated was then dissolved in HOAc $(12 \mathrm{~mL}$ ), and zinc (nano-size activated powder, $99.9 \%, 800 \mathrm{mg}$ ) was added. After stirring for $6 \mathrm{~h}$ at $30^{\circ} \mathrm{C}$, TLC 
(20:1 EtOAc-MeOH) showed that the starting material was completely consumed. The reaction mixture was passed through a short silica gel column (eluent: ethyl acetate), the combined eluent was concentrated, and the residue was again acetylated with $\mathrm{Ac}_{2} \mathrm{O}$ in pyridine. The acetylated product was then dissolved in $\mathrm{MeOH}$ containing $\mathrm{Pd} / \mathrm{C}$ ( $10 \% \mathrm{Pd}, 50 \mathrm{mg}$ ), and $\mathrm{H}_{2}$ was bubbled into the mixture at $\mathrm{rt}$ for about $12 \mathrm{~h}$. The reaction mixture was filtered, and the filtrate was concentrated to dryness. Treatment of the residue with $\mathrm{Ac}_{2} \mathrm{O}$ in pyridine as described above gave a syrup that was subjected to silica gel column chromatography (1:1 petroleum ether-EtOAc) to afford $16(230 \mathrm{mg}, 71 \%)$ as a foamy solid: $[\alpha]_{\mathrm{D}}^{25}$ +17 (c 2, $\left.\mathrm{CHCl}_{3}\right) ;{ }^{1} \mathrm{H}$ NMR $\left(500 \mathrm{MHz}, \mathrm{CDCl}_{3}\right): \delta 6.97,6.89(2 \mathrm{~d}$, $\left.2 \times 2 \mathrm{H}, J 9.0 \mathrm{~Hz}, \mathrm{CH}_{3} \mathrm{OC}_{6} \mathrm{H}_{4} \mathrm{O}\right), 5.99(\mathrm{~d}, 1 \mathrm{H}, J 7.5 \mathrm{~Hz}), 5.80(\mathrm{dd}, 1 \mathrm{H}$, $J$ 3.5, $13.5 \mathrm{~Hz}$ ), 5.69 (d, 1H, J 7.5 Hz), $5.46(\mathrm{~d}, 1 \mathrm{H}, J 3.5 \mathrm{~Hz}), 5.42$ (dd, $1 \mathrm{H}, J 3.5,11.0 \mathrm{~Hz}), 5.38(\mathrm{~d}, 1 \mathrm{H}, J 3.5 \mathrm{~Hz}), 5.36(\mathrm{~d}, 1 \mathrm{H}, J$ $4.0 \mathrm{~Hz}$ ), 5.31 (dd, 1H, J 3.0, $11.0 \mathrm{~Hz}$ ), 5.30 (d, 1H, J $4.0 \mathrm{~Hz}$ ), 5.25 $(\mathrm{d}, 1 \mathrm{H}, J 3.0 \mathrm{~Hz}), 5.01-5.14(\mathrm{~m}, 7 \mathrm{H}), 4.56-4.58(\mathrm{~m}, 1 \mathrm{H}), 4.38-4.41$ $(\mathrm{m}, 2 \mathrm{H}), 4.18-4.21(\mathrm{~m}, 2 \mathrm{H}), 4.03-4.15(\mathrm{~m}, 7 \mathrm{H}), 3.98(\mathrm{t}, 1 \mathrm{H}, J$ $8.5 \mathrm{~Hz}), 3.90(\mathrm{t}, 1 \mathrm{H}, J 7.0 \mathrm{~Hz}), 3.83-3.86(\mathrm{~m}, 1 \mathrm{H}), 3.72-3.80(\mathrm{~m}$, $5 \mathrm{H}), 3.42-3.47(\mathrm{~m}, 2 \mathrm{H}), 1.88-2.16(\mathrm{~m}, 45 \mathrm{H}, 15 \mathrm{Ac}), 1.89(\mathrm{~s}, 3 \mathrm{H})$. ${ }^{13} \mathrm{C}$ NMR $\left(125 \mathrm{MHz}, \mathrm{CDCl}_{3}\right): \delta 171.2,171.0,170.6,170.5,170.4$, $170.3,170.2,170.1,170.0,169.8,155.5,150.4,117.6,114.9,99.6$, 99.2, 99.1, 95.8, 94.6, 77.3, 77.0, 76.7, 73.8, 73.6, 72.4, 71.7, 71.5, $71.1,70.7,69.7,68.3,68.2,67.4,67.3,66.7,66.4,66.2,65.3,64.7$, 63.0, 61.7, 61.3, 55.7, 53.3, 23.3, 23.1, 21.0, 20.7, 20.6, 20.5, 15.8, 14.1. MALDI-TOFMS calcd for $\mathrm{C}_{67} \mathrm{H}_{90} \mathrm{~N}_{2} \mathrm{O}_{39}: 1456.5[\mathrm{M}]^{+}$; found, $1569.7[\mathrm{M}+\mathrm{Na}]^{+}$. Anal. Calcd for $\mathrm{C}_{67} \mathrm{H}_{90} \mathrm{~N}_{2} \mathrm{O}_{39}$ : C, 52.00; $\mathrm{H}, 5.86$. Found: C, 52.17; H, 5.96.

1.11. 4-Methoxyphenyl 2-acetamido-2-deoxy- $\beta$-D-galactopyranosyl-( $1 \rightarrow 4)$ - $\alpha$-D-galactopyranosyl- $(1 \rightarrow 3)$-2-acetamido-2-deoxy$\beta$-D-galactopyranosyl- $(1 \rightarrow 6)$-[ $\alpha$-L-fucopyranosyl- $(1 \rightarrow 2)]-\beta$-Dgalactopyranoside (1)

To a solution of $16(232 \mathrm{mg}, 0.15 \mathrm{mmol})$ in $\mathrm{MeOH}(10 \mathrm{~mL})$ was added $1 \mathrm{M}$ NaOMe until the $\mathrm{pH}$ of the solution reached 9-10. The reaction mixture was allowed to stir at $\mathrm{rt}$ for $10 \mathrm{~h}$, then neutralized with Dowex-50 $\left(\mathrm{H}^{+}\right)$ion exchange resin. The mixture was filtered, the filtrate was concentrated, and the resulting residue was purified by a Bio-Gel P2 column using $\mathrm{H}_{2} \mathrm{O}$ as eluent. The desired fractions were combined and freeze dried to afford compound $1(138 \mathrm{mg}$, $92 \%)$ as an amorphous solid: $[\alpha]_{\mathrm{D}}^{25}+5\left(\mathrm{c} 0.4, \mathrm{H}_{2} \mathrm{O}\right) ;{ }^{1} \mathrm{H}$ NMR $\left(500 \mathrm{MHz}, \mathrm{D}_{2} \mathrm{O}\right): \delta 7.08,6.99\left(2 \mathrm{~d}, 2 \times 2 \mathrm{H}, J 9.0 \mathrm{~Hz}, \mathrm{CH}_{3} \mathrm{OC}_{6} \mathrm{H}_{4} \mathrm{O}\right)$, $5.27\left(\mathrm{~s}, 1 \mathrm{H}, \mathrm{H}-1^{\mathrm{I}}\right), 5.19\left(\mathrm{~d}, 1 \mathrm{H}, J 7.5 \mathrm{~Hz}, \mathrm{H}-1^{\mathrm{II}}\right), 5.06$ (d, $1 \mathrm{H}, J 4.0 \mathrm{~Hz}$, $\left.\mathrm{H}-1^{\mathrm{IV}}\right), 4.59\left(\mathrm{~d}, 1 \mathrm{H}, J 8.5 \mathrm{~Hz}, \mathrm{H}-1^{\mathrm{III}}\right), 4.53\left(\mathrm{~d}, 1 \mathrm{H}, J 8.5 \mathrm{~Hz}, \mathrm{H}-1^{\mathrm{V}}\right)$, $3.58-4.21(\mathrm{~m}, 33 \mathrm{H}), 2.05,1.75(2 \mathrm{~s}, 6 \mathrm{H}, 2 \mathrm{Ac}), 1.11(\mathrm{~s}, 3 \mathrm{H}, \mathrm{H}-6 \mathrm{l}) .{ }^{\mathrm{I}} \mathrm{C}$ NMR $\left(125 \mathrm{MHz}, \mathrm{D}_{2} \mathrm{O}\right): \delta 175.8,174.9,155.2,151.5,118.6,118.1$, $116.0,103.7,101.8,100.7,100.0,96.3,78.0,77.5,76.7,75.6,75.5$, $74.5,74.0,72.5,71.6,71.3,70.2,69.9,69.6,69.2,69.1,69.0,68.5$ 67.7, 64.8, 61.8, 61.7, 60.8, 56.6, 53.3, 51.3, 23.1, 22.7, 20.8, 16.0. MALDI-TOFMS calcd for $\mathrm{C}_{41} \mathrm{H}_{64} \mathrm{~N}_{2} \mathrm{O}_{26}: 1000.4[\mathrm{M}]^{+}$; found, 1023.7 $[\mathrm{M}+\mathrm{Na}]^{+}$. Anal. Calcd for $\mathrm{C}_{41} \mathrm{H}_{64} \mathrm{~N}_{2} \mathrm{O}_{26}$ : C, 49.20; H, 6.44. Found: $\mathrm{C}$, $49.01 ; \mathrm{H}, 6.52$.

\section{Acknowledgments}

This work was supported by NNSF projects of China(20621703, 20872172, 20732001) and Project 2009ZX09501-011.

\section{Supplementary data}

Supplementary data associated with this article can be found, in the online version, at doi:10.1016/j.carres.2010.07.025.

\section{References}

1. Orskov, F.; Orskov, I. Methods Microbiolol. 1984, 14, 43-112.

2. Raetz, C. R.; Whitefield, C. Annu. Rev. Biochem. 2002, 71, 635-700.

3. Nataro, J. P.; Kaper, J. B. Clin. Microbiol. Rev. 1998, 11, 142-201.

4. Sengupta, P.; Bhattacharyya, T.; Shashkov, A. S.; Kochanowski, H.; Basu, S. Carbohydr. Res. 1995, 277, 283-290.

5. Kaiser, J. Science 2004, 305, 460.

6. Turnbull, J. E.; Field, R. A. Nat. Chem. Biol. 2007, 3, 74-77.

7. Seeberger, P. H.; Werz, D. B. Nature 2007, 446, 1046-1051.

8. Li, M.; Liu, X.; Shao, J.; Shen, J.; Jia, Q.; Yi, W.; Song, J. K.; Woodward, R.; Chow, C. S.; Wang, P. G. Biochemistry 2008, 47, 378-387.

9. Yang, F.; He, H.; Du, Y.; Lu, M. Carbohydr. Res. 2002, 337, 1165-1169.

10. Kanaya, T.; Yagi, S.; Schweizer, F.; Takeda, T.; Kiuchi, F.; Hada, N. Chem. Pharm. Bull. 2010, 58, 811-817.

11. Cheng, L.; Chen, Q.; Liu, J.; Du, Y. Carbohydr. Res. 2007, 342, 975-981.

12. Hanessian, S. Preparative Carbohydrate Chemistry; Marcel Dekker: New York, 1997.

13. Kihlberg, J.; Eichler, E.; Bundle, D. R. Carbohydr. Res. 1991, 211, 59-75.

14. Knapp, S.; Nandan, S. R. J. Org. Chem. 1994, 59, 281-283.

15. Leigh, D. A.; Smart, J. P.; Truscello, A. M. Carbohydr. Res. 1995, 276, 417-424.

16. Schmidt, R. R. Toepfer, A. Tetrahedron Lett. 1991, 32, 3353-3356.

17. Imamura, A.; Ando, H.; Ishida, H.; Kiso, M. Org. Lett. 2005, 7, 4415-4418.

18. Smid, P.; de Ruiter, G. A.; van der Marel, G. A.; Rombouts, F. M.; van Boom, J. H. J. Carbohydr. Chem. 1991, 10, 833-849.

19. Greene, T. W.; Wuts, P. G. M. Protecting Groups in Organic Synthesis; Wiley: New York, 1999; 510-511.

20. Zeng, Y.; Kong, F. Carbohydr. Res. 2003, 338, 2047-2056. 